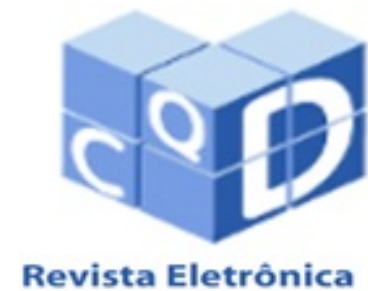

Revista Eletrônica

Paulista de Matemática

ISSN 2316-9664

Volume 12, jul. 2018

\section{Christian José Santos}

\section{Gonçalves}

Universidade Estadual de

Maringá - DMA - UEM

chrisssgoncalves@gmail.com

\title{
Construção do anel de polinômios em uma indeterminada utilizando módulos
}

Construction of the ring of polynomials in one indeterminate using modules

\section{Resumo}

O conceito de módulos sobre um anel comutativo com unidade $A$ é uma generalização da noção de espaço vetorial. Como em álgebra linear, é possível definir conjunto linearmente independente, base e dimensão de maneira bem semelhante. O objetivo deste artigo é construir o anel de polinômios $A[X]$. Ele será gerado como $A$-módulo pelo conjunto linearmente independente $\left\{1, X, X^{2}, \ldots\right\}$ de elementos de $S(A)$, conjunto das sequências em $A$.

Palavras-chave: Anéis de polinômios. Módulos. Sequências quase nulas.

Renan Willian Prado

Universidade Estadual de Campinas - Imecc - Unicamp renanwillianprado@gmail.com

\section{Abstract}

The concept of modules over a commutative ring with identity $A$ is a generalization of the notion of vector space. As in linear algebra, it is possible to define linearly independent set, base and dimension in a very similar way. The objective of this article is construct the ring of polynomials $A[X]$. It will be generated as $A$ module by the linearly independent set $\left\{1, X, X^{2}, \ldots\right\}$ of elements of $S(A)$, set of the sequences in $A$.

Keywords: Ring of polynomials. Modules. Sequences almost null. 


\section{Introdução}

É fato que em toda a história da matemática, os primeiros conceitos sobre polinômios, sobretudo as equações polinomiais se deram antes mesmo do formalismo matemático moderno. Um polinômio é uma expressão da forma $a_{n} x^{n}+a_{n-1} x^{n-1}+\cdots+a_{1} x+a_{0}$, onde os coeficientes $a_{n}, a_{n-1}, \ldots a_{0}$ são elementos de um anel comutativo com unidade e o $x$ é chamado de indeterminada. Nosso objetivo será formalizar este conceito, assim como fez Peano com os números naturais no século XIX, por exemplo.

O estudo de anéis se originou a partir de duas importantes classes, a classe dos anéis de polinômios em $n$ variáveis sobre o corpo dos números complexos, e a classe dos anéis de inteiros algébricos de um corpo de números algébricos. Essas duas classes são responsáveis por vários resultados, muito sofisticados e um tanto quanto elegantes.

Neste artigo, precisaremos somente de alguns conceitos básicos de módulos, na qual introduziremos na primeira seção. Para um estudo mais aprofundado veja [1] ou [2].

Na seção seguinte, o conjunto $S(A)$ das sequências de elementos em $A$, será ainda um anel comutativo com unidade. O subconjunto $X^{\mathbb{N}}:=\left\{X^{n} \in S(A) \mid n \in \mathbb{N}\right\}$ de elementos de $S(A)$ definido pelas potências de $X=\left(x_{n}\right)$ tal que $x_{n}=0$, se $n \neq 1$ e $x_{n}=1$, se $n=1$, será um conjunto linearmente independente, assim, definiremos $A[X]$ como sendo o conjunto gerado por $X^{\mathbb{N}}$. Tal sequência $X$, é chamada de indeterminada.

É possível construir o anel de polinômios apenas com o conceito de sequências quase nulas como feito em [2] e [3], ou de modo mais intuitivo, como em [4]. No entanto, a construção feita aqui é mais algébrica do que em termos de sequências, um conceito mais analítico.

\section{A-módulos}

Definição 1. Seja $A=(A,+, \cdot)$ um anel comutativo com unidade e $M=(M, \oplus)$ um grupo comutativo. Dizemos que $M$ é um A-modulo se existe uma operação adicional, $\odot: A \times M \rightarrow M$, tal que dados $a_{1}, a_{2} \in A$ e dados $m_{1}, m_{2} \in M$ vale

- $1 \odot m_{1}=m_{1}$,

- $\left(a_{1} \cdot a_{2}\right) \odot m_{1}=a_{1} \odot\left(a_{2} \odot m_{1}\right)$,

- $\left(a_{1}+a_{2}\right) \odot m_{1}=a_{1} \odot m_{1} \oplus a_{2} \odot m_{1}$;

- $a_{1} \odot\left(m_{1} \oplus m_{2}\right)=a_{1} \odot m_{1} \oplus a_{1} \oplus m_{2}$.

Para simplificar, daqui em diante escreveremos $a_{1} \odot m_{1}=a_{1} m_{1}$ e $m_{1} \oplus m_{2}=m_{1}+m_{2}$.

Definição 2. Seja A um anel e $M$ um A-módulo. Um subgrupo $N$ de $M$ é um A-submódulo, se a multiplicação por escalar do módulo M preserva $N$, isto é, se

$$
a \odot n \in N, \forall a \in A e \forall n \in N .
$$

Abaixo segue dois exemplos clásicos de $A$-módulos. Estes exemplos mostram que $A$-módulos generalizam a noção de espaço vetorial e que nem todos os $A$-módulos são espaços vetoriais.

Exemplo 3. Seja $K$ um corpo e $V$ um espaço vetorial. Então $V$ é um $K$-módulo, onde $\odot$ é o produto por escalar e $V$ é grupo abeliano com a soma $\oplus$ de vetores. 
Exemplo 4. Todo grupo $G=(G,+)$ comutativo é um $\mathbb{Z}$ módulo dotado da operação $\odot: \mathbb{Z} \times G \rightarrow$ $G \operatorname{com} n \odot g=n g \equiv \sum_{i=1}^{n} g$.

Motivado pelos conceitos em espaços vetoriais, podemos definir um subconjunto gerado por um subconjunto $S$, subconjunto linearmente independente e base.

Definição 5. Seja $S \subset M, M$ um A-módulo, então o gerado por $S$ é o conjunto $(S)$ tal que

$$
(S)=\left\{v \in M \mid \exists\left\{a_{s^{\prime}}\right\}_{s^{\prime} \in S^{\prime}} \subset A, v=\sum_{s^{\prime} \in S^{\prime}} a_{s^{\prime}} s^{\prime} \text { e } S^{\prime} \subset S \text { é finito }\right\} .
$$

Quando $(A)=M$, diremos que $M$ é gerado por $S$ ou que $S$ é um gerador de $M$ e quando $S$ é finito diremos que $M$ é finitamente gerado, ou quando se quer explicitar $S$, diremos que $M$ é finitamente gerado por $S$. Além disso, se $v \in S$ diremos que v é combinação linear de elementos de $S$.

Claramente $(S)$ é um submódulo de $M$, assim temos a mesma noção que em espaços vetoriais onde o gerado por um subconjunto também é subespaço.

Definição 6. Seja $S$ um subconjunto de $M$, um A-módulo, dizemos que S é linearmente independente se para qualquer família finita $\left\{s_{i}^{\prime}\right\}_{i \in I}$ de elementos de $S$ e qualquer família $\left\{a_{i}\right\}_{i \in I}$ de elementos de A vale que

$$
\sum_{i \in I} a_{i} s_{i}=0 \Rightarrow a_{i}=0, \forall i \in I
$$

Exemplo 7. Todo subconjunto S linearmente independente de $V$, um $K$ espaço vetorial, é linearmente independente de vendo $V$ como $K$-módulo.

Definição 8. Uma base $S$ de um A-módulo $M$, é um subconjunto linearmente independente e gerador de M. Nesse caso, diremos que Mé um A-módulo livre. Chamamos a cardinalidade de $S$, denotamos por $|S|=\# S$, um posto de $M$ ou uma dimensão de $M$.

O Lema abaixo será essencial para demonstrar que a dimensão de um $A$-módulo está bem definido.

Lema 9. Seja $M$ um A-módulo e I um ideal de A. Então $I M=\left\{\sum_{j=1}^{n} i_{j} m_{j} \mid i_{j} \in I\right.$ e $m_{j} \in M, \forall 1 \leq$ $j \leq n\}$ é um A-módulo e M/IM é um A/I-módulo segundo a operação:

$$
\odot: \begin{array}{cll}
A / I \times M / I M & \longrightarrow & M / I M \\
(\lambda+I, m+I M) & \longmapsto & \lambda m+I M .
\end{array}
$$

Demonstração. Temos inicialmente que

$$
I M=\left\{\sum_{j=1}^{n} i_{j} m_{j} \mid i_{j} \in I \text { e } m_{j} \in M, \forall 1 \leq j \leq n\right\} .
$$

Temos que $I M$ é fechado para soma por um oposto, pois dados dois elementos $x, y \in I M$ temos que $x=\sum_{i=1}^{n} i_{j} m_{j} \mathrm{e}-y=-\sum_{i=n+1}^{n+m} i_{j} m_{j}=\sum_{i=n+1}^{n+m}\left(-i_{j}\right) m_{j}$ e assim $x-y=\sum_{i=1}^{n} i_{j} m_{j}+$ $\sum_{i=n+1}^{n+m}\left(-i_{j}\right) m_{j}=\sum_{i=1}^{n+m} \operatorname{sign}(n-j) i_{j} m_{j}$. Portanto $I M$ é também grupo abeliano. Além disso, dado $a \in A$, vale que $a x=a\left(\sum_{i=1}^{n} i_{j} m_{j}\right)=\sum_{i=1}^{n} a\left(i_{j} m_{j}\right)=\sum_{i=1}^{n}\left(a i_{j}\right) m_{j}$, mas para cada $j$, com $1 \leq j \leq n$, temos $a i_{j} \in I$, pois $I$ é um ideal de $A$. Logo vale que a operação $\left.\odot\right|_{A \times I M}$ tem imagem contida em $I M, \operatorname{logo} I M$ é, também, um $A$-módulo. 
Temos que se $\left(\lambda_{1}+I, m_{1}+I M\right)=\left(\lambda_{2}+I, m_{2}+I M\right)$, então $\lambda_{1}-\lambda_{2} \in I$ e $m_{1}-m_{2} \in I M$. Assim, como $\lambda_{1} m_{1}-\lambda_{2} m_{2}=\lambda_{1} m_{1}-\lambda_{1} m_{2}+\lambda_{1} m_{2}-\lambda_{2} m_{2}=\lambda_{1}\left(m_{1}-m_{2}\right)+\left(\lambda_{1}-\lambda_{2}\right) m_{2}$, então como cada parcela está em $I M$, temos que a soma também está. Assim, $M / I M$ é um $A / I$-módulo.

Proposição 10. Sejam $S$ e $S^{\prime}$ duas bases de $M$, um A-módulo, então $|S|=\left|S^{\prime}\right|$, isto é, o posto é único e está bem definido.

Demonstração. Seja $I$ um ideal maximal de $A$, que existe pelo lema de Zorn, e seja $Z$ qualquer base de $M$, um $A$-módulo.

Considere $\{z+I M\}_{z \in Z}$. Temos que é base de $M / I M$, um $A / I$-módulo, e $|Z|=\left|\{z+I M\}_{z \in Z}\right|$. De fato, dada $\{z+I M\}_{z \in Z_{i}}$ uma família finita de elementos de $\{z+I M\}_{z \in Z}$ e $\left\{a_{z}+I\right\}_{z \in Z_{i}}$ também uma família finita de elementos de $A / I$, se

$$
\sum_{z \in Z_{i}}\left(\alpha_{z}+I\right)(z+I M)=0+I M
$$

então $\sum_{z \in Z_{i}} \alpha_{z} z \in I M$. Logo, como $Z$ é base de $M$ e reagrupando, caso necessário, existe um segundo subconjunto finito $Z_{j}$ de $Z$ e existem $i_{z} \in I \forall z \in Z_{j}$ tal que ainda vale

$$
\sum_{z \in Z_{i}} \alpha_{z} z=\sum_{z \in Z_{j}} i_{z} z
$$

Assim, cada $\alpha_{z} \in I$, com $z \in Z_{i}$. Temos portanto, $\alpha_{z}+I=0+I$ para todo $z \in Z_{i}$.

Mostraremos que $\{z+I M\}_{z \in Z}$ gera $M / I M$. De fato, seja $m \in M$, temos que existe subconjunto finito $Z_{k}$ de $Z$, por $Z$ ser base de $M$, e elementos $a_{z} \in A$ para todo $z \in Z_{k}$, tal que

$$
m=\sum_{z \in Z_{k}} a_{z} z
$$

Assim, $m+I M=\left(\sum_{z \in Z_{k}} a_{z} z\right)+I M=\sum_{z \in Z_{k}}\left(a_{z} z+I M\right)=\sum_{z \in Z_{k}}\left(a_{z}+I\right)(z+I M)$.

Falta mostrar que $|Z|=\{z+I M\}_{z \in Z}$, mas isso é claro, pois, como $\{z+I M\}_{z \in Z}$ é base de $M / I M$, se $z \neq z^{\prime}$, então $z+I M \neq z^{\prime}+I M$. Logo $|Z|=\left|\{z+I M\}_{z \in Z}\right|$.

Como $I$ é ideal maximal, temos que $A / I$ é corpo, assim $M / I M$ é corpo sobre $A / I$, segue, portanto, que toda base de $M / I M$ tem a mesma cardinalidade. Assim, finalmente, dadas duas bases $S$ e $S^{\prime}$ de um $A$-módulo $M$, segue que, pelos comentários anteriores, $\left|S^{\prime}\right|=\left|\left\{s^{\prime}+I M\right\}_{s^{\prime} \in S^{\prime}}\right|=$ $\left|\{s+I M\}_{s \in S}\right|=|S|$, como queríamos demonstrar.

Abaixo daremos um exemplo de um módulo $M$ que não é livre, isto é, um módulo que não possui base.

Exemplo 11. Seja $n>1$ um número inteiro, o grupo abeliano $\mathbb{Z}_{n}$ não é um $\mathbb{Z}$-módulo livre. Apesar de ser, claramente, um $\mathbb{Z}$-módulo, $\mathbb{Z}_{n}, n>1$, não é livre. De fato, dado $\bar{z} \in \mathbb{Z}_{n}, n \cdot \bar{z}=\overline{0}$. Logo nenhum subconjunto $S$ de $\mathbb{Z}_{n}$ pode ser linearmente independente sobre $\mathbb{Z}$.

A proposição seguinte diz que, como em espaços vetoriais, que todo elemento é escrito de modo único como combinação linear de vetores da base.

Proposição 12. Seja $S \subset M$ uma base para $M$. Todo elemento de $M$ se escreve, de modo único, como combinação linear não nula de elementos de $S$, i.e., dados $X$ e $Y$, dois pares de subconjuntos finitos de $S,\left\{a_{x}\right\}_{x \in X} e\left\{b_{y}\right\}_{y \in Y}$, dois subconjuntos finitos de elementos não nulos de A, tais que $\sum_{x \in X} a_{x} x=\sum_{y \in Y} b_{y} y$, então $X=Y e\left\{a_{x}\right\}_{x \in X}=\left\{b_{y}\right\}_{y \in Y}$. 
Demonstração. Dados $X$ e $Y,\left\{a_{x}\right\}_{x \in X}$ e $\left\{b_{y}\right\}_{y \in Y}$ tais como o enunciado, então $\sum_{x \in X} a_{x} x=$ $\sum_{y \in Y} b_{y} y$. Logo,

$$
\sum_{x \in X} a_{x} x-\sum_{y \in Y} b_{y} y=0
$$

Dado $x^{*} \in X$, suponha, por absurdo, que $x^{*}$ não está em $Y$, então, $\left(\sum_{x \in X-\left\{x^{*}\right\}} a_{x} x-\sum_{y \in Y} b_{y} y\right)+$ $a_{x^{*}} X^{*}=0$. Como $\sum_{x \in X-\left\{x^{*}\right\}} a_{x} x-\sum_{y \in Y} b_{y} y \in\left(\left(X-\left\{x^{*}\right\}\right) \cup Y\right)$, i.e., está no espaço gerado por $\left(X-\left\{x^{*}\right\}\right) \cup Y$, segue que existe uma família finita $\left\{c_{z}\right\}_{z \in\left(X-\left\{x^{*}\right\}\right) \cup Y}$ de elementos de $A$ tal que $\sum_{x \in X-\left\{x^{*}\right\}} a_{x} x-\sum_{y \in Y} b_{y} y=\sum_{z \in\left(X-\left\{x^{*}\right\}\right) \cup Y} c_{z} z$. Logo,

$$
\sum_{z \in\left(X-\left\{x^{*}\right\}\right) \cup Y} c_{z} z+a_{x^{*}} x^{*}=0 .
$$

Assim, necessariamente $a_{x^{*}}=0$, o que é um absurdo. Da mesma forma, se prova que dado $y^{*} \in Y$, então $y^{*} \in X$. Temos, portanto, que $X=Y$. Agora como $X=Y$,

$$
\sum_{x \in X} a_{x} x-\sum_{y \in Y} b_{y} y=\sum_{x \in X}\left(a_{x}-b_{x}\right) x=0
$$

Assim, $a_{x}=b_{x}, \forall x \in X$, donde $\left\{a_{x}\right\}_{x \in X}=\left\{b_{y}\right\}_{y \in Y}$, como queríamos demonstrar.

Finalmente temos todas as ferramentas necessárias para se definir polinômios em uma indeterminada.

\section{Polinômios em uma indeterminada}

Seja um anel $A$ comutativo com unidade e considere o conjunto $S(A)$ das sequências com elementos em $A$, isto é,

$$
S(A)=\{s \subset \mathbb{N} \times A \mid s \text { é função }\} .
$$

Denotaremos $s$, um elemento de $S(A)$, por $\left(s_{n}\right)_{n}$ para dizer que $s(n)=s_{n}$ para todo $n \in \mathbb{N}$.

Definição 13. Em $S(A)$ consideremos duas operações $\oplus e \odot$, chamadas de adição e produto por escalar respectivamente, da seguinte forma: $\forall f, g \in S(A)$ e $a \in A$,

$$
\begin{aligned}
& \oplus: S(A) \times S(A) \rightarrow S(A) \\
& \odot: A \times S(A) \rightarrow S(A) \\
& (f, g) \longmapsto f \oplus g \\
& (a, f) \longmapsto a \odot f
\end{aligned}
$$

$\operatorname{Com}(f \oplus g)_{n}=\left(f_{n}+g_{n}\right)_{n} e(a \odot f)_{n}=\left(a f_{n}\right)$.

Em algumas momentos usaremos as notações $f+g$ e $a f$ para as operações $f \oplus g$ e $a \odot f$ respectivamente.

Proposição 14. Com as operações $\oplus e \odot$ definidas acima segue que $S(A)$ é um A-módulo.

Demonstração. De fato, é fácil provar que $S(A)$ é grupo comutativo com a operação $\oplus$. Falta mostrar que $\forall a, b \in A$ e $\forall f, g \in S(A)$, vale as condições da definição (1):

- $1 f=\left(1 f_{n}\right)_{n}=\left(f_{n}\right)_{n}=f$,

- $(a b) f=\left((a b) f_{n}\right)_{n}=\left(a\left(b f_{n}\right)\right)_{n}=a\left(b f_{n}\right)_{n}=a(b f)$, 
- $(a+b) f=\left((a+b) f_{n}\right)_{n}=\left(a f_{n}+b f_{n}\right)_{n}=\left(a f_{n}\right)_{n}+\left(b f_{n}\right)_{n}=a\left(f_{n}\right)_{n}+b\left(f_{n}\right)_{n}=a f+b f$,

- $a(f+g)=a\left(f_{n}+g_{n}\right)_{n}=\left(a\left(f_{n}+g_{n}\right)\right)_{n}=\left(a f_{n}+a g_{n}\right)=\left(a f_{n}\right)_{n}+\left(a g_{n}\right)_{n}=a\left(f_{n}\right)_{n}+a\left(g_{n}\right)_{n}=$ $a f+a f$.

Nosso próximo passo será fazer com que $S(A)$ passe a ser um anel comutativo com unidade, para isso precisaremos definir uma segunda operação em $S(A)$ :

Definição 15. Em $S(A)$ definimos $\circ: S(A) \times S(A) \rightarrow S(A)$ tal que se $f$ e g estão em $S(A)$, então $f \circ g=c$, onde

$$
c_{n}=\sum_{i=0}^{n} f_{i} g_{n-i}, \forall n \in \mathbb{N} .
$$

Denotamos $f \circ g$ por $f g$, isto é, $f \circ g=f g$.

Proposição 16. $S(A)=(S(A), \oplus, \circ)$ é um anel comutativo com unidade.

Demonstração. Já sabemos que $S(A)$ é grupo comutativo com a operação $\oplus$ e portanto falta mostrar que existe $1 \in S(A)$ tal que para quaisquer $f, g, h \in S(A)$ vale

1. $1 \circ f=f$,

2. $f \circ g=g \circ f$,

3. $(f \circ g) \circ h=f \circ(g \circ h)$,

4. $f \circ(g+h)=f \circ g+f \circ h$.

Tome $1=\left(s_{n}\right)_{n}, s_{n}=1$, se $n=0$, e $s_{n}=0$, caso contrário. Temos que para quaisquer $f, g, h \mathrm{em}$ $S(A)$ vale

1. $1 \circ f=f$. De fato, $1 f=\left(s_{n}\right)_{n}\left(f_{n}\right)_{n}=\left(\sum_{j=0}^{n} s_{j} f_{n-j}\right)_{n}=\left(s_{0} f_{n}\right)_{n}=\left(1 f_{n}\right)_{n}=\left(f_{n}\right)=f$. Da mesma forma, $f 1=\left(\sum_{j=0}^{n} f_{j} s_{n-j}\right)_{n}=\left(f_{n} s_{n-n}\right)=\left(f_{n} 1\right)_{n}=\left(f_{n}\right)_{n}=f$,

2. $f \circ g=g \circ f$. De fato, temos que $f \circ g=\left(f_{n}\right)_{n}\left(g_{n}\right)_{n}=\left(\sum_{j=0}^{n} f_{j} g_{n-j}\right)_{n}=\left(\sum_{j=0}^{n} f_{n-j} g_{j}\right)_{n}=$ $\left(\sum_{j=0}^{n} g_{j} f_{n-j}\right)_{n}=\left(g_{n}\right)_{n}\left(f_{n}\right)_{n}=g \circ f$,

3. $(f \circ g) \circ h=f \circ(g \circ h)$. Temos que

$$
\begin{aligned}
(f g) h & =\left(\sum_{i=0}^{n} f_{i} g_{n-i}\right)_{n} h=\left(\sum_{i=0}^{n} f_{i} g_{n-i}\right)_{n}\left(h_{n}\right)_{n}= \\
& =\left(\sum_{j=0}^{n}\left(\sum_{i=0}^{j} f_{i} g_{j-i}\right) h_{n-j}\right)_{n}=\left(\sum_{j=0}^{n} \sum_{i=0}^{j} f_{i} g_{j-i} h_{n-j}\right)_{n}= \\
& =\left(\sum_{i=0}^{n} \sum_{j=i}^{n} f_{i} g_{j-i} h_{n-j}\right)_{n}= \\
& =\left(\sum_{i=0}^{n} \sum_{j=i}^{n} f_{i} g_{j-i} h_{n-j}\right)_{n}=\left(\sum_{i=0}^{n} f_{i}\left(\sum_{j=i}^{n} g_{j-i} h_{n-j}\right)\right)_{n}= \\
& =\left(\sum_{i=0}^{n} f_{i}\left(\sum_{k=0}^{n-i} g_{k} h_{n-i-k}\right)\right)_{n}=\left(f_{n}\right)_{n}\left(\sum_{k=0}^{n} g_{k} h_{n-k}\right)_{n}= \\
& =\left(f_{n}\right)_{n}\left(\left(g_{n}\right)_{n}\left(h_{n}\right)_{n}\right)=f(g h) .
\end{aligned}
$$


Aqui vale a pena fazer um comentário do que foi feito. Note que $\{(i, j) \in \mathbb{Z} \times \mathbb{Z} \mid 0 \leq j \leq$ $n, 0 \leq i \leq j\}=\{(i, j) \in \mathbb{Z} \times \mathbb{Z} \mid 0 \leq i \leq n, i \leq j \leq n\}$, fazendo uma mudança de coordenadas tal como feita em Cálculo ao trocar a ordem de integração da integral dupla. Por essa razão $\sum_{j=0}^{n} \sum_{i=0}^{j} a_{i, j}=\sum_{i=0}^{n} \sum_{j=i}^{n} a_{i, j}$, pela comutatividade da operação adição.

4. $f \circ(g+h)=f \circ g+f \circ h$. De fato,

$$
\begin{aligned}
f(g+h)=\left(f_{n}\right)_{n}\left(\left(g_{n}\right)+\left(h_{n}\right)_{n}\right) & =\left(f_{n}\right)_{n}\left(\left(g_{n}+h_{n}\right)_{n}\right)=\left(\sum_{i=0}^{n} f_{i}\left(g_{n-i}+h_{n-i}\right)\right)_{n}= \\
& \left.=\left(\sum_{i=0}^{n} f_{i} g_{n-i}+f_{i} h_{n-i}\right)\right)_{n}=\left(\sum_{i=0}^{n} f_{i} g_{n-i}+\sum_{i=0}^{n} f_{i} h_{n-i}\right)_{n}= \\
& =\left(\sum_{i=0}^{n} f_{i} g_{n-i}\right)_{n}+\left(\sum_{i=0}^{n} f_{i} h_{n-i}\right)_{n}= \\
& =\left(f_{n}\right)_{n}\left(g_{n}\right)+\left(f_{n}\right)_{n}\left(h_{n}\right)_{n}=f g+f h .
\end{aligned}
$$

Portanto $S(A)$ é anel com as operações dadas, como queríamos demonstrar.

Definição 17. A sequência $X=\left(x_{n}\right)$ tal que $x_{n}=0$, se $n \neq 1$ e $x_{n}=1$, se $n=1$, é chamada indeterminada.

Definição 18. Para manter a uniformidade daqui em diante, defina $X^{0}=1$, onde $1 \in S(A)$ é a unidade deste anel.

Definição 19. Defina, indutivamente, para cada $n \geq 1, X^{n+1}=X X^{n}$.

A proposição seguinte descreve como é a lei da sequência $X^{n}$ e será importante nas próximas proposições e demonstrações.

Proposição 20. Dado $n \geq 1$, vale $X^{n}=\left(\delta_{m, n}\right)_{m}$, onde $\delta_{m, n}$ é a função delta de Kronecker, definida por, $\delta_{m, n}=0$, se $m \neq n$ e $\delta_{m, n}=1$, se $m=n$.

Demonstração. A lei, por definição, vale para $n=1$. Assim, suponha por indução que a lei valha para $n$, qualquer natural dado, temos que $X^{n+1}=X X^{n}=\left(x_{m}\right)_{m}\left(\delta_{m, n}\right)_{m}=\left(\sum_{i=0}^{m} x_{i} \delta_{m-i, n}\right)_{m}=$ $\left(x_{1} \delta_{m-1, n}\right)_{m}=\left(1 \delta_{m-1, n}\right)_{m}=\left(\delta_{m-1, n}\right)_{m}=\left(\delta_{m, n+1}\right)_{m}$, como queríamos demonstrar.

Abaixo vamos provar que vale a regra da soma da potência da indeterminada.

Proposição 21. Dados $i, j \in \mathbb{N}, X^{i} X^{j}=X^{i+j}$.

Demonstração. Dados $i, j \in \mathbb{N}$, temos que

$$
\begin{gathered}
X^{i} X^{j}=\left(\delta_{n, i}\right)_{n}\left(\delta_{n, j}\right)_{n}=\left(\sum_{k=1}^{n} \delta_{k, i} \delta_{n-k, j}\right)_{n}=\left(\delta_{i, i} \delta_{n-i, j}\right)_{n} \\
=\left(\delta_{n-i, j}\right)_{n}=\left(\delta_{n, i+j}\right)_{n}=X^{i+j}
\end{gathered}
$$

como queríamos demonstrar.

Proposição 22. O subconjunto de elementos de $S(A)$ dado por $\left\{X^{n} \in S(A) \mid n \in \mathbb{N}\right\}$ é um conjunto linearmente independente de $S(A)$. 
Demonstração. Seja $\left\{X^{n}\right\}_{n \in \eta}$ uma família finita de elementos de $\left\{X^{n} \in S(A) \mid n \in \mathbb{N}\right\}$ e seja $\left\{a_{n}\right\}_{n \in \eta}$ uma família finita de elementos de $A$ tal que $\sum_{n \in \eta} a_{n} X^{n}=0$. Claramente, $\eta \subset \mathbb{N}$ é finito. Pela Proposição 21, $\sum_{n \in \eta} a_{n} X^{n}=\sum_{n \in \eta} a_{n}\left(\delta_{m, n}\right)_{m}=\sum_{n \in \eta}\left(a_{n} \delta_{m, n}\right)_{m}=\left(\sum_{n \in \eta} a_{n} \delta_{m, n}\right)_{m}=$ 0. Logo,

$$
\sum_{n \in \eta} a_{n} \delta_{m, n}=0, \forall m \in \mathbb{N}
$$

Seja $n^{\prime} \in \eta$, por $1, \sum_{n \in \eta} a_{n} \delta_{n^{\prime}, n}=0$, assim $\sum_{n \in \eta} a_{n} \delta_{n^{\prime}, n}=a_{n}^{\prime} \delta_{n^{\prime}, n^{\prime}}=a_{n}^{\prime}=0$, como queríamos demonstrar.

A seguir vem a principal definição do presente artigo, onde a definição vem de modo natural.

Definição 23. O conjunto gerado, veja Definição 5, por $X^{\mathbb{N}}=\left\{X^{n} \in S(A) \mid n \in \mathbb{N}\right\}$ denotado por $A[X]$, isto é, $A[X]=\left(\left\{X^{n} \in S(A) \mid n \in \mathbb{N}\right\}\right)=\left\{\sum_{x \in X^{*}} a_{x} x \mid\left\{a_{x}\right\}_{x \in X^{*}} \subset A\right.$ com $X^{*} \subset X^{\mathbb{N}}$ finito $\}$ éo conjunto dos polinômios com coeficiente em $A$. $O$ conjunto $A[X]$ é chamado anel dos polinômios em uma indeterminada.

Segue que, como o esperado, pela Proposição 22, o conjunto $X^{\mathbb{N}}$ é base para o anel dos polinômios. Assim, os polinômios tem dimensão infinita enumerável e, pela Proposição 10, todas as bases tem dimensão infinita enumerável. Além disso, note que dado $x \in X^{\mathbb{N}}$, existe um único $i \in \mathbb{N}$ tal que $x=X^{i}$, assim podemos escrever as somas $\sum_{x \in X^{*}} a_{x} x$ por $\sum_{i \in \eta} a_{i}^{\prime} X^{i}$, onde $\eta$ é um subconjunto finito dos naturais e $a_{x}=a_{i}{ }_{i}$, quando $x=X^{i}$. Além disso, como $\eta$ é finito segue que $\eta$ é limitado, digamos por $n \in \mathbb{N}$, $\operatorname{logo}$ defina $b_{i}=0$, se $i \leq n$ e $i \notin \eta$ e $b_{i}=a^{\prime}{ }_{i}$, se $i \leq n$ e $i \in \eta$. Portanto, $\sum_{x \in X^{*}} a_{x} x=\sum_{i \in \eta} a_{i}^{\prime} X^{i}=\sum_{i=0}^{n} b_{i} X^{i}$. Assim, os polinômios tem a forma usual de se escrever. Por essa razão, em geral, denotamos um polinômio por $P(X)$.

Definição 24. Seja $P(X)=\sum_{i=0}^{n} a_{i} X^{i} \in A[X]$ um polinômio não nulo. Dizemos que um número $\operatorname{gr}(P(X)) \in \mathbb{N}$ é o grau do polinômio $P(X)$, se $P(X)=\sum_{i=1}^{g r(P(X))} a_{i} X^{i}$ e $a_{g r(P(X))} \neq 0$, isto é, $\operatorname{gr}(P(X))$ é o maior $n$, tal que $a_{n}$ não é nulo.

Definição 25. Diz-se que uma sequência $\left(s_{n}\right)_{n \in \mathbb{N}} \in S(A)$ é quase nula se existe $n_{0} \in \mathbb{N}$ tal que $s_{n}=0, \forall n \geq n_{0}$ ou, equivalentemente, o conjunto $\left\{n \in \mathbb{N} \mid s_{n} \neq 0\right\}$ é finito. O conjunto dessas sequências denotaremos por $E$.

Proposição 26. O conjunto dos polinômios em uma indeterminada é o conjunto das sequências quase nulas.

Demonstração. Seja $P(X)=\sum_{i=0}^{n} a_{i} X^{i} \in A[X]$ um polinômio. Pela Proposição 20,

$$
P(X)=\sum_{i=0}^{n} a_{i} X^{i}=\sum_{i=0}^{n} a_{i}\left(\delta_{m, i}\right)_{m}=\sum_{i=0}^{n}\left(a_{i} \delta_{m, i}\right)_{m}=\left(\sum_{i=0}^{n} a_{i} \delta_{m, i}\right)_{m} .
$$

Tome $m \geq n+1$, para que $\delta_{m, i}=0$, para todo $0 \leq i \leq n, \operatorname{logo} \sum_{i=0}^{n} a_{i} \delta_{m, i}=\sum_{i=0}^{n} a_{i} 0=0$ e assim $P(X) \in E$. Logo $A[X] \subset E$. Seja $\left(s_{n}\right)_{n} \in E$, temos que existe $n_{0} \in \mathbb{N}$ tal que $s_{n}=0, \forall n \geq n_{0}$, mas assim temos que $\left(s_{n}\right)_{n}=\sum_{i=0}^{n} s_{i}\left(\delta_{m, i}\right)_{m}=\sum_{i=0}^{n} s_{i} X^{i}$. Portanto, $\left(s_{n}\right)_{n} \in A[X]$. Logo $E \subset A[X]$. Donde $A[X]=E$.

Abaixo daremos uma aplicação imediata da proposição acima.

Corolário 27. O conjunto das sequências quase nulas nos racionais é enumerável. 
Demonstração. Pela Proposição 26, temos que as sequências quase nulas são exatamente os polinômios com coeficientes racionais. Como $\mathbb{Q}[X]$ tem base enumerável e os racionais são enumeráveis, segue que o o conjunto das sequências é enumerável.

A definição de polinômios, como gerado por $X^{\mathbb{N}}$, permite provar facilmente que produtos de polinômios é também um polinômio, como demonstrado na proposição seguinte.

Proposição 28. Dados $P(X), Q(X) \in A[X]$, então $P(X) Q(X) \in A[X]$.

Demonstração. Dados $P(X), Q(X)$, existe duas famílias finitas de elementos de $A$, digamos, $\left\{a_{i}\right\}_{0 \leq i \leq m}$ e $\left\{b_{j}\right\}_{0 \leq j \leq n}$, tais que $P(X)=\sum_{i=0}^{m} a_{i} X^{i}$ e $Q(X)=\sum_{j=0}^{n} b_{j} X^{j}, \log \mathrm{o}$

$$
P(X) Q(X)=\left(\sum_{i=0}^{m} a_{i} X^{i}\right)\left(\sum_{j=0}^{n} b_{j} X^{j}\right)=\sum_{i=0}^{m} \sum_{j=0}^{n} a_{i} b_{j} X^{i} X^{j}=\sum_{i=0}^{m} \sum_{j=0}^{n} a_{i} b_{j} X^{i+j} .
$$

Assim, $P(X) Q(X)$ está no gerado por $X^{\mathbb{N}}$ e portanto está em $A[X]$.

Até agora não está claro o motivo de se ter tomado um polinômio como uma sequência infinita e não como uma função $f: A \rightarrow A$ tal que $f(X)=\sum_{i=1}^{n} a_{i} X^{i}$. A importância de tal construção vai ser mostrada no exemplo a seguir.

Exemplo 29. Seja $n \in \mathbb{N}$ e considere $\mathbb{Z}_{n}$ o conjunto dos inteiros módulo $n$. Seja $f: \mathbb{Z}_{n} \rightarrow \mathbb{Z}_{n}$ tal que $f(X)=\prod_{i=1}^{n}(X-\bar{i})$. Temos que $f(X)=\overline{0}$ para todo $X \in \mathbb{Z}_{n}$, isto é, a função $f$ é a função identicamente nula. No entanto, o polinômio $\prod_{i=1}^{n}(X-\bar{i}) \in S(A)$ não é, claramente, polinômio nulo.

O exemplo acima nos motiva a definir o valor de $f(X)$ aplicado num elemento de $A$.

Definição 30. Seja $f(X)=\sum_{i=0}^{n} a_{i} X^{i}$ um polinômio em $A[X]$. Chamamos de valor do polinômio $f(X)$ em $x \in A$, denotado por $f(x)$, o escalar $f(x) \in A$ tal que $f(x)=\sum_{i=0}^{n} a_{i} x^{i}$. Quando $f(x)=0$, chamamos $x$ de uma raiz ou um zero do polinômio $f$.

Note que, como a representação de um polinômio é única, pois $X^{\mathbb{N}}$ é base, pela Proposição 22, e pela unicidade da combinação linear, vide Proposição 12, dado um polinômio $P(X) \in A[x]$ e $x \in A$, existe um único valor $P(x) \in A$. Isso nos permite definir função polinomial:

Definição 31. Chamamos de função polinomial qualquer função $f: A \rightarrow A \operatorname{com} f(\alpha)=P(\alpha)$ para todo $\alpha \in A$ com $P(X) \in A[X]$. Quando $f(\alpha)=0$, chamamos $\alpha$ de uma raiz ou um zero do polinômio $P(X)$. Dizemos que, nesse caso, $f$ é uma função polinomial determinada pelo polinômio $P(X)$.

\section{Conclusão}

É possível construir o anel de polinômios apenas com o conceito de sequências quase nulas como feito em [2] e [3], ou de modo mais intuitivo, como em [4]. No entanto, a construção feita aqui é mais algébrica do que em termos de sequências, um conceito mais analítico. Além disso, nossa construção é bem mais geral, por exemplo, pode-se definir uma família finita elementos de um $A$-módulo $M$, digamos $\left\{X_{i}\right\}_{1 \leq i \leq n}$ que comutam no produto de vetores, a fim de definir o conjunto polinômios de $n$ indeterminadas como sendo o gerado pelo conjunto linearmente independente $\left\{\prod_{i=1}^{n} X_{i}^{\alpha_{i}} \mid \alpha_{i} \in \mathbb{N}, \forall 1 \leq i \leq n\right\}$. Temos portanto uma grande liberdade de construção de estruturas somente com a teoria básica de $A$-módulos.

GONÇALVES, C. J. S.; PRADO, R. S. Construção do anel de polinômios em uma indeterminada utilizando módulos. C.Q.D.- Revista Eletrônica Paulista de Matemática, Bauru, v. 12, p. 47-56, jul. 2018.

DOI: $10.21167 /$ cqdvol12201823169664cjsgrwp4756 Disponível em: http://www.fc.unesp.br/\#!/departamentos/matematica/revista-cqd/ 


\section{Referências}

[1] ATIYAH, M. F.; MACDONALD, I. G. Introduction to commutative algebra. London: Addison-Wesley Publishing Co., 1969.

[2] GARCIA, A.; LEQUAIN, Y. Elementos de álgebra. 5. ed. Rio de Janeiro: Instituto Nacional de Matematica Pura e Aplicada, 2008.

[3] MONTEIRO, L. H. J. Elementos de álgebra. Rio de Janeiro: IMPA, 1969.

[4] FRALEIGH, J. B. A first course in abstract algebra. 7. ed. Boston. Pearson Education, Inc., 2003.

[5] HOFFMAN, K.; KUNZE, R. Álgebra linear. São Paulo: Editora Polígono, 1971. 